\title{
Genome-Wide Association Mapping of diverse set of spring wheat germplasm in Western Siberia
}

\author{
Shepelev S.S. ${ }^{*}$, Shamanin V.P. ${ }^{1}$, Pototskaya I.V. ${ }^{1}$, Pozherukova V.E. ${ }^{1}$, Chursin A.S. ${ }^{1}$, \\ Morgounov A.I. ${ }^{2}$ \\ ${ }^{1}$ Omsk State Agrarian University, Omsk, Russia \\ ${ }^{2}$ CIMMYT-Turkey, Ankara, Turkey \\ *e-mail:sergeyschepelew@mail.ru
}

The research object was the set of 150 accessions, which comprises: CIMMYT synthetic wheat lines with Ae. tauschii genome (44 accessions); synthetic lines from Kyoto University (14); spring bread wheat varieties from the USA and Canada (15); varieties and lines of Omsk State Agrarian University (18), varieties and lines of Omsk ARI (17); wheat varieties of network KASIB-18 from Russia and Kazakhstan (42). The SNPs with MAF less than $5 \%$ and missing data more than $20 \%$ were removed from the analysis. After filtering, 46.268 SNP markers of 143 accessions were used for genetic diversity analysis. The GBS derived SNPs were well distributed across the 21 chromosomes. The B genome had the highest number of SNPs $(17.309, \sim 37 \%)$, followed by the A genome (15.776, 34\%), and the D genome (13.183, 29\%). There were 1.258 SNPs located in scaffolds that are not anchored to any of the chromosomes. The number of SNPs per chromosome ranged from 808 (4D) to 3.148 (2B). A total 143 accessions were evaluated on 35 economically valuable traits under field conditions in Omsk in 2017 and 2018 for genome-wide association study. GWAS detected 243 MTAs distributed across 21 chromosomes for traits with phenotypic variance explained (PVE) ranging from $0.3 \%$ to $25.0 \%$. The highest number of MTAs was detected in the A genome (72), the B genome (65), and the D genome (62). Thus, for the first time in the Russian Federation genome-wide association study under conditions of Western Siberia was carried out. Were identified 243 MTAs associated with 35 economically valuable traits. Some of identified MTAs were reliable in both research years, and had a pleiotropic effect on 2-4 traits at the same time. The markers associated with these traits were identified. 